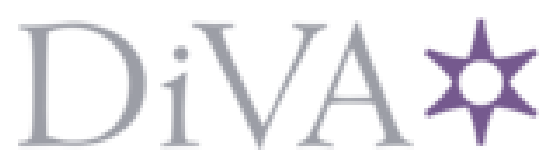

http://www.diva-portal.org

This is the published version of a paper presented at IEEE International Conference on Electronics, Computing and Communication Technologies (CONECCT), 17-19 Jan. 2013,Bangalore.

Citation for the original published paper:

Yajnanarayana, V., Dwivedi,, S., De Angelis, A., Händel, P. (2013)

Design of impulse radio UWB transmitter for short range communications using PPM signals. In: IEEE International Conference on Electronics, Computing and Communication Technologies (CONECCT), 2013 (pp. 1-4). IEEE conference proceedings http://dx.doi.org/10.1109/CONECCT.2013.6469303

N.B. When citing this work, cite the original published paper.

(C) 2013 IEEE. Personal use of this material is permitted. Permission from IEEE must be obtained for all other uses, in any current or future media, including reprinting/republishing this material for advertising or promotional purposes, creating new collective works, for resale or redistribution to servers or lists, or reuse of any copyrighted component of this work in other works.

Permanent link to this version:

http://urn.kb.se/resolve?urn=urn:nbn:se:kth:diva-1 19858 


\title{
Design of impulse radio UWB transmitter for short range communications using PPM signals
}

\author{
Vijaya Yajnanarayana, Satyam Dwivedi, Alessio De Angelis, Peter Händel \\ ACCESS Linnaeus Centre, \\ Signal Processing Lab, \\ KTH Royal Institute of Technology, Stockholm, Sweden \\ email:\{vpy,dwivedi,ales,ph\}@kth.se
}

\begin{abstract}
There are several practical challenges in designing an ultra wideband (UWB) device for communication. From the physical layer perspective, signaling technique should be optimally designed to work in synergy with the underneath hardware to achieve maximum performance. In this paper we propose a new cost effective hardware architecture for UWB communication and propose a variant of pulse position modulation (PPM) method which achieves maximum bit rate under the practical constraints imposed by UWB hardware.
\end{abstract}

Index terms: Ultra wideband, UWB communication, pulse position modulation, time to digital converter (TDC), peak to average power (PAPR).

\section{INTRODUCTION}

In UWB communication system a low power message signal is spread in to a wideband. The transmitted power will be as low as in the order of $0.5 \mathrm{~mW}$ and the bandwidth is of greater than $500 \mathrm{MHz}$. Regulatory authorities like Federal Communications Commission (FCC) define the UWB emission using fractional bandwidth. Refer [1] for these definitions.

UWB technology provides several advantages for communication. The well known Shannon capacity theorm is given by

$$
C=B \log \left(1+\frac{S}{N}\right)
$$

where $C$ denotes capacity, $B$ denotes bandwidth and $S / N$ denotes signal to noise ratio (SNR). Since spectrum occupied by UWB signal is significantly large, UWB has potential to communicate with very high data rates.

The wide bandwidth ensures good frequency diversity and makes multipaths easily resolvable which are beneficial from the communication system design [2]. However UWB systems has to co-exist with the other narrowband technologies. The compliance requirements from regulatory bodies for UWB system are very stringent to ensure that it does not interfere with the existing narrowband systems. This makes the design of UWB for communication very challenging. These requirements are generally specified via spectral masks. Figure 1 shows the FCC mandated spectral mask for UWB

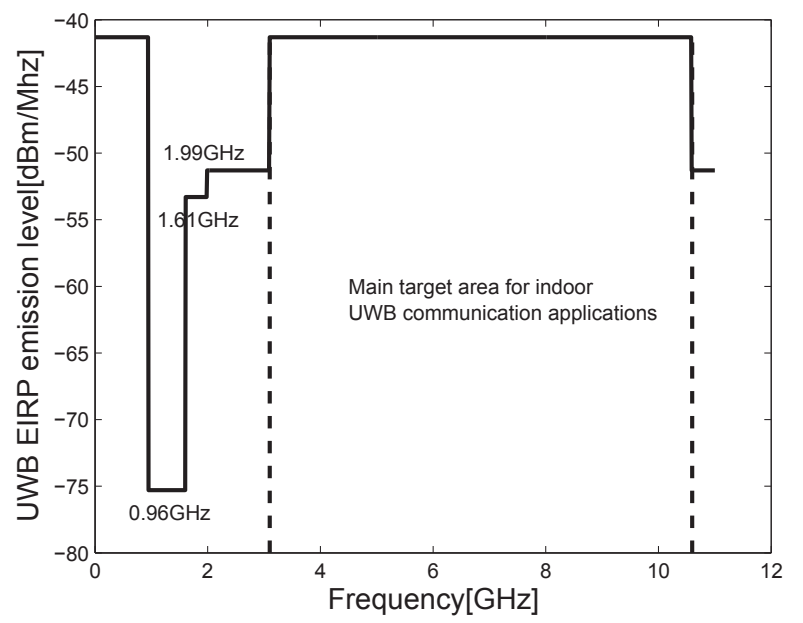

Fig. 1. Spectral mask specified by FCC for indoor UWB system [3].

system.

There are several ways to spread the low power message signal in to wide band without interfering with other spectrum users. The pulse based methods are popular because of the ease of implementation in the hardware, together with interesting properties which can be exploited from pulse based communication to optimally exploit the nature of the wide band available.

Wide range of pulse shapes have been explored for the UWB communication from rectangular to Gaussian [4]. Gaussian pulses and their derivatives, usually called monopulses are effective due to the easy construction and good resolution in both time and frequency. Several modulation techniques are proposed for UWB systems using these pulses. Primarily they are variants of PPM, binary phase shift keying (BPSK) or onoff keying (OOK) [5][6].

The remainder of this paper is organized as follows. We start with Section II, here we explain the properties of pulses used for UWB system, Then we will look at the UWB Hardware and the constraints it imposes on the pulse 
generation. In Section III, we discuss the UWB transmitter hardware and design challenges. Then in Section IV, we will derive an optimal performance criterion for high throughput communication given the constraints of the hardware and yet balancing the requirements of regulatory bodies. In Section V, we demonstrate the performance archived by the design and finally, Section VI, details the conclusions from the design and results demonstrated.

\section{Pulse COnstruction for UWB Transmitters}

There are many UWB shapes explored in literature, however the one based on Gaussian pulses makes a good choice for building a cost effective UWB transceiver. The reasoning for this is that a simple transistor switching "on" and "off" to generate a narrow pulse will form this shape instead of a rectangular shape due to imperfections in micro-electronic design [3].

The Gaussian pulse equation with mean $\mu$ and variance $\sigma^{2}$ can be written as

$$
\frac{1}{2 \pi \sigma^{2}} e^{\frac{-(t-\mu)^{2}}{2 \sigma^{2}}}
$$

More useful form of this equation for system design is defined in [3]. This is a scaled version of (2) with mean $\mu=0$ and $\tau^{2}=2 \pi \sigma^{2}$. This form is shown as

$$
p(t)=-e^{-2 \pi\left(\frac{t}{\tau}\right)^{2}}
$$

In a typical UWB device, when the signal passes through the UWB antenna, it will have a differentiation effect on the signal. Similar effect is observed when the receiver receives the pulses. The first and second order Gaussian pulses are given by

$$
\begin{gathered}
p^{\prime}(t)=\frac{-4 \pi t}{\tau^{2}} e^{\frac{-2 \pi t^{2}}{\tau^{2}}} \\
p^{\prime \prime}(t)=-4 \pi e^{\frac{-2 \pi t^{2}}{\tau^{2}}}\left(\frac{-\tau^{2}+4 \pi t^{2}}{\tau^{4}}\right)
\end{gathered}
$$

For analytical and simulation analysis we have used the 2nd order Gaussian pulses shown in (5). As shown in Figure 1 the operating point of interest is greater than $3 \mathrm{GHz}$, hence from the mask perspective average equivalent isotropically radiated power (EIRP) should be less than $-41.3 \mathrm{dBm} / \mathrm{MHz}$ in this region. Figure 2 shows the simple Gaussian pulse (5) of width $T=1$ ns with $\tau=0.3$ ns and Figure 3 shows the power spectral density (PSD) of the pulse.

Train of these pulses can be used for UWB communication, The PSD of these pulses is shown in Figure 4, Notice here a significant peak to average power ratio (PAPR) due to the comb formations. This results in inefficient usage of power, and causes severe impact on the range UWB device can support while still honoring the mask constraints described in Figure 1.

One way to overcome this problem is to completely randomize pulse intervals. We created a train of pulses with pulse

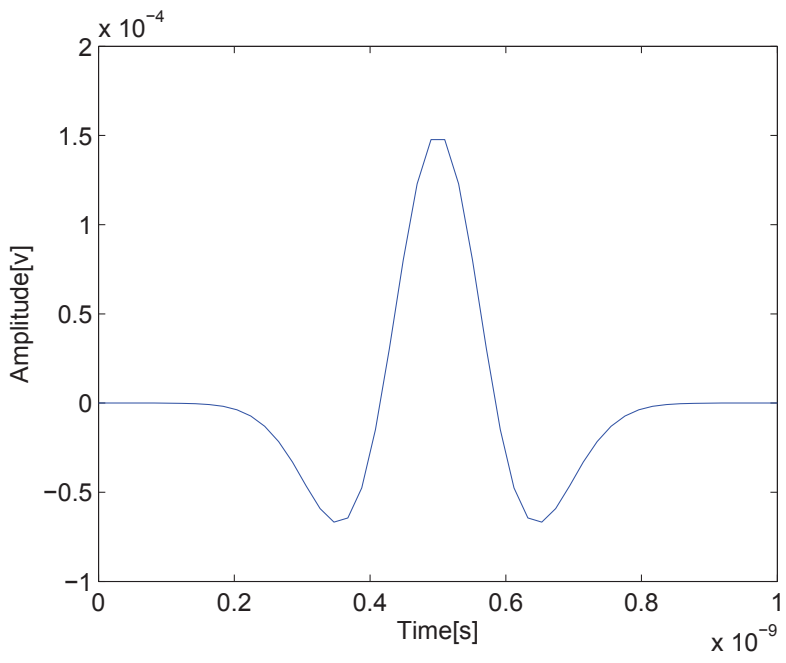

Fig. 2. Monopulse with $T=1 \mathrm{~ns}$ and $\tau=0.3 \mathrm{~ns}$.

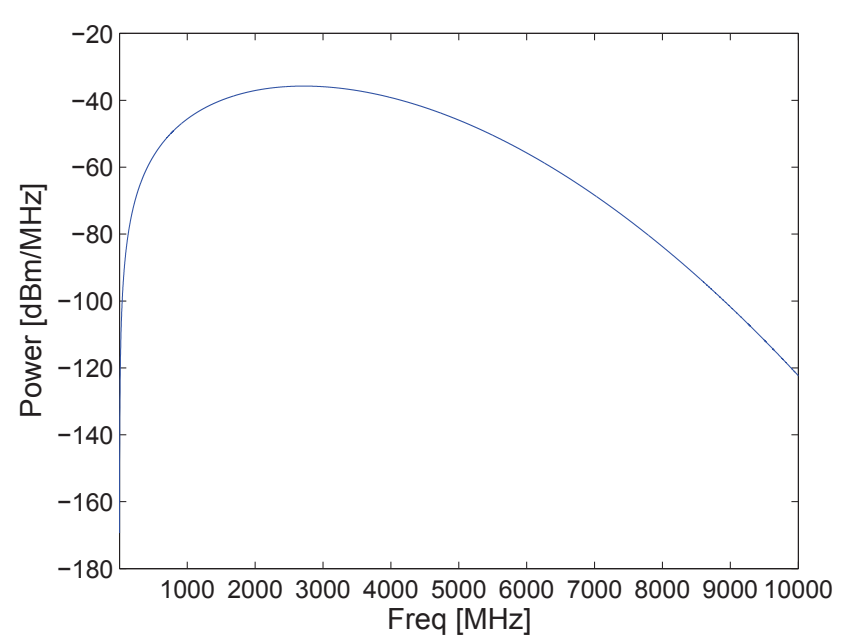

Fig. 3. PSD of monopulse with $T=1 \mathrm{~ns}$ and $\tau=0.3 \mathrm{~ns}$.

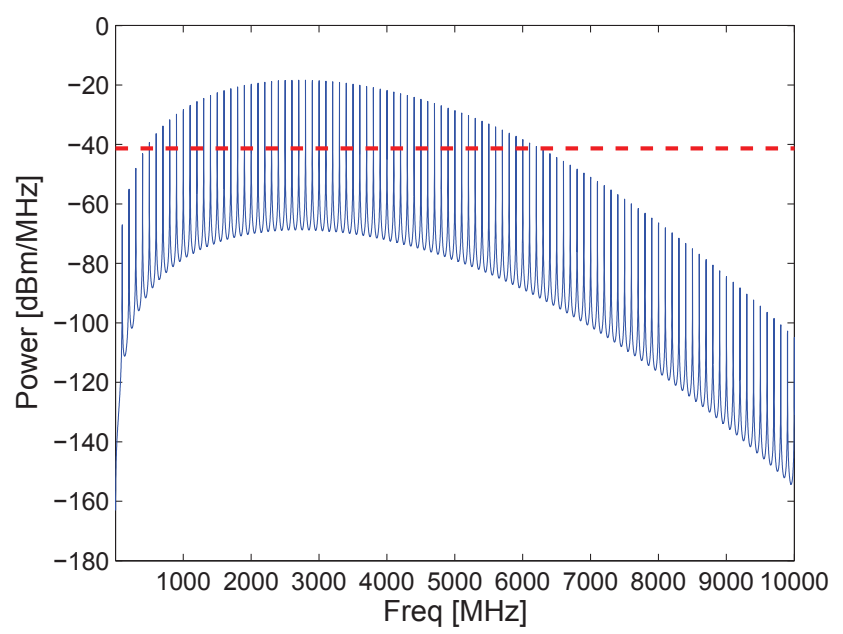

Fig. 4. PSD of $1 \mathrm{~ns}$ pulses with pulse repetition period $T_{\mathrm{PRT}}=10 \mathrm{~ns}$. 


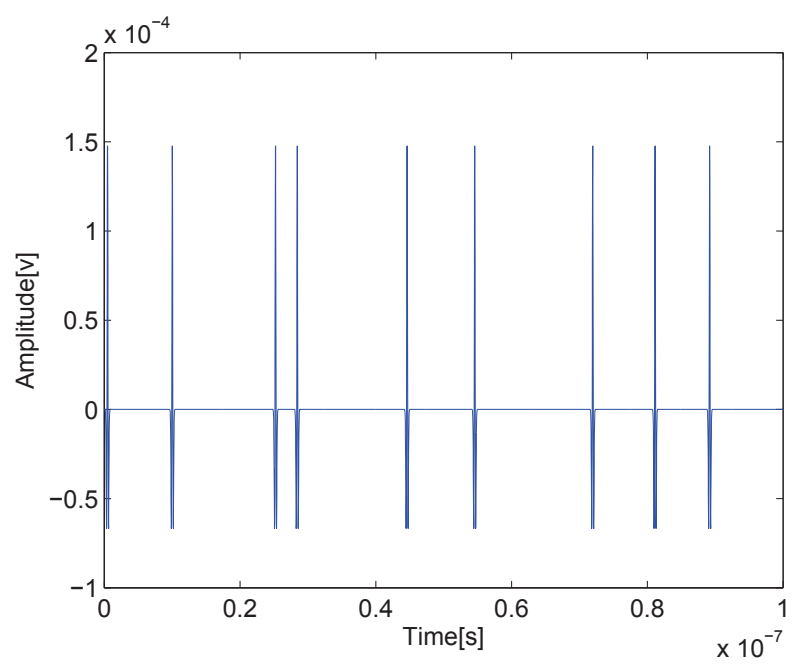

Fig. 5. Train of pulses with randomized $T_{\mathrm{PRT}}$.

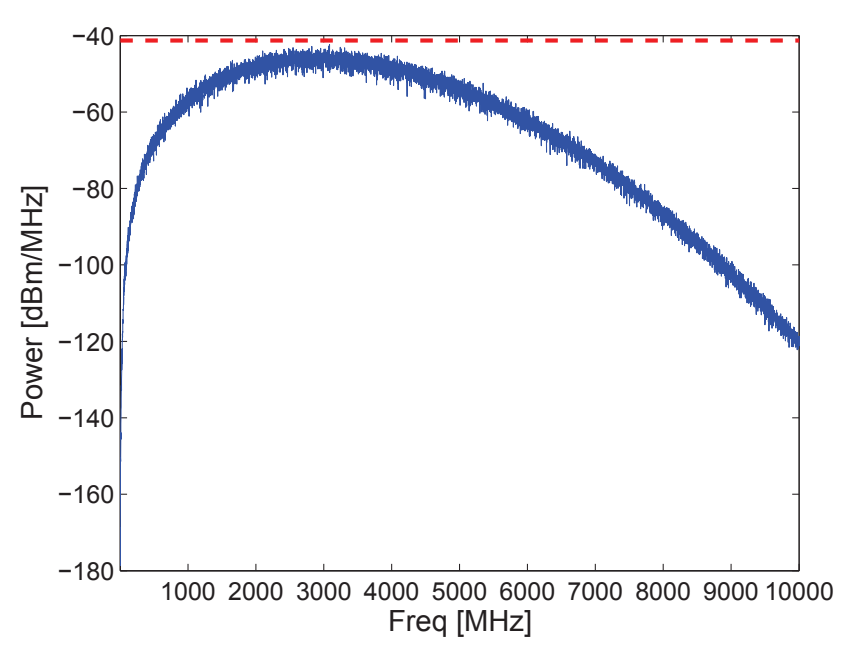

Fig. 6. PSD of pulses with randomized $T_{\mathrm{PRT}}$.

interval $T_{\mathrm{PRT}}$ as discrete uniform random variable with mean $10 \mathrm{~ns}$. The probabability mass function (PMF) of $T_{\mathrm{PRT}}$ is

$$
\operatorname{PMF}\left(T_{\mathrm{PRT}}\right)= \begin{cases}\frac{1}{n} & \text { If } T \leq T_{\mathrm{PRT}} \leq 20 \mathrm{~ns} . \\ 0 & \text { Otherwise. }\end{cases}
$$

Here $T$ is the pulse width of the Gaussian pulse and $n$ is the number of bins / discretized-intervals. $T_{\mathrm{PRT}}$ is varied from $T$ instead of 0 to avoid collision between pulses. The train of these pulses is shown in Figure 5. With this modification the comb formations are eliminated and PAPR (Figure 6) is almost zero and thus power can be more effectively managed within the mask constraints. Notice in Figure 6 that PSD is below $-41.3 \mathrm{dBm} / \mathrm{MHz}$ as mandated by $\mathrm{FCC}$ for the same pulse power used in the periodic case.

\section{UWB HARDWARE}

The sensor architecture for the UWB hardware is shown in Figure 7. This hardware consists of analog UWB measurement

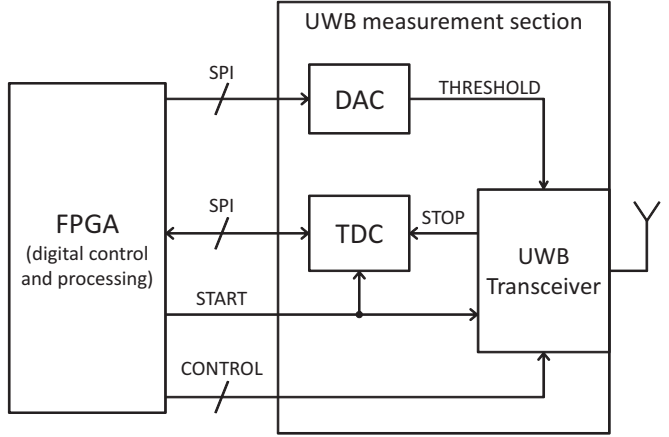

Fig. 7. UWB Sensor architecture [7].

section, designed in-house. It has a digital processing section based on FPGA [7]. This architecture is based on time to digital conversion (TDC), which was originally designed for ranging round trip time calculations. However the same can be used to measure the time between the pulse intervals, thereby enabling demodulation of PPM signals and thus can be used for UWB communication as well. Further details of this UWB sensor hardware architecture can be found in [7].

\section{Modulation Parameter optimization}

The TDC in the sensor architecture measures the time between pulses [7]. This has a fixed resolution, which will limit the granularity of time measurements that can be done using the TDC, the modulation index $\Delta$ of any pulse position modulation scheme cannot be less than this resolution. Also there should be minimum separation $\left(T_{\mathrm{ms}}\right)$ between the pulses due to the limitation from the step recovery diode used in the pulse generation module [7]. These two critical constraints needs to be addressed when designing the pulse trains. We propose a PPM variant signaling technique which can address these two critical issues and choose optimal modulation order to maximize bit rate.

If we assume $T_{\mathrm{ms}}$ to be the minimum separation between the pulses and $\Delta$ as the modulation index of the PPM, then the pulse period $T_{\mathrm{S}}$ and bit rate $R_{b}$ using this variant of the PPM signalling are given by

$$
\begin{aligned}
T_{\mathrm{S}} & =T_{\mathrm{ms}}+\Delta M \\
R_{b} & =\frac{\log _{2} M}{T_{\mathrm{ms}}+\Delta M}
\end{aligned}
$$

where $\log _{2} M$ is the modulation order. From UWB hardware design the values for $T_{\mathrm{ms}}$ and $\Delta$ are fixed, they come from the two UWB hardware constraints discussed above. We try to optimally pick modulation parameter $M$. To do this we evaluate (8) choose that $M$ that maximizes the bit rate $R_{b}$. The variation of $R_{b} \mathrm{v} / \mathrm{s} M$ is shown in the Figure 8 . We choose $T_{\mathrm{ms}}=10 \mathrm{~ns}$ and $\Delta=1 \mathrm{~ns}$ as typical parameters for 


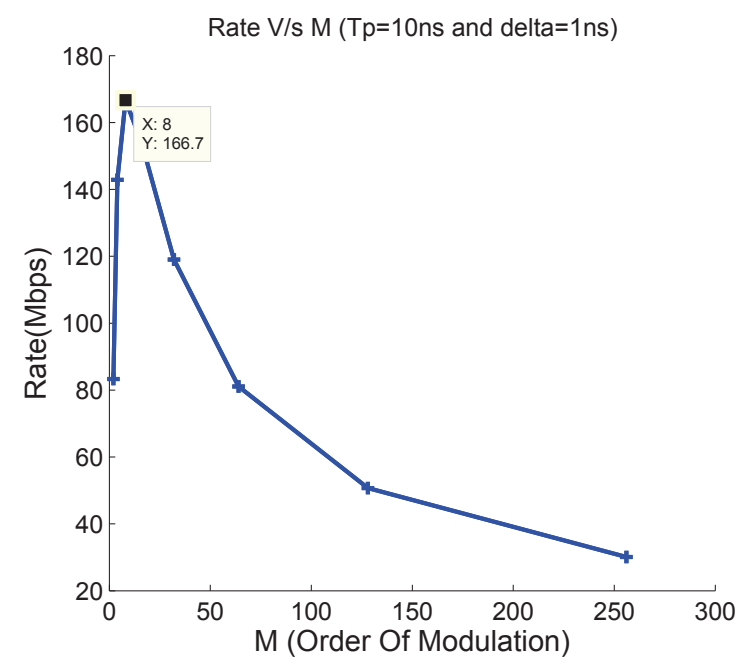

Fig. 8. Bit Rate V/S Modulation Order

the hardware constraints. Notice that for $T_{\mathrm{ms}}=10 \mathrm{~ns}$ and $\Delta=1 \mathrm{~ns}$ bit rate peaks at $M=8$.

\section{PERformance ANALYSis}

We generated a variant of M-PPM signal with $M=8$ which always ensures a minimum gap of $T_{\mathrm{ms}}=10 \mathrm{~ns}$ and uses a modulation index of $\Delta=1 \mathrm{~ns}$. This signal is shown in Figure 9. Notice in Figure 9 a gap of 10 ns between pulses and 3 bits of information is PPM modulated in the remaining $8 \mathrm{~ns}$ (since $\Delta=1 \mathrm{~ns}, M=8$ ) this achieves maximum bit rate under the hardware constraints discussed in the previous section. Though we cannot achieve as good performance as Figure 6 because now pulse train has deterministic gaps between pulses $\left(T_{\mathrm{ms}}\right)$ and the pulse positions are quantized $(\Delta)$ for it to work with chosen UWB hardware. However since the data is assumed random it does smoothen the PSD. The PSD of this PPM variant signalling formulation is shown in the Figure 10.

\section{CONCLUSION}

In this paper we analyzed the behavior of the train of Gaussian monopulses. We concluded that the PAPR can be reduced by varying the pulse positions randomly. This enables optimal power utilization in UWB hardware. We proposed a TDC based architecture for UWB communication and proposed a modulator for it. Here we arrived at an optimal $M$ for a PPM variant signaling to achieve maximum bit rate under the constraints of UWB transceiver architecture.

\section{ACKNOWLEDGEMENTS}

Parts of the work have been funded by The Swedish Agency for Innovation Systems (VINNOVA).

\section{REFERENCES}

[1] "First report and order, revision of part 15 of the commision's rules regarding ultra-wideband transmission systems," tech. rep., FCC, Washington DC, ET Docket 98-153, 2002.

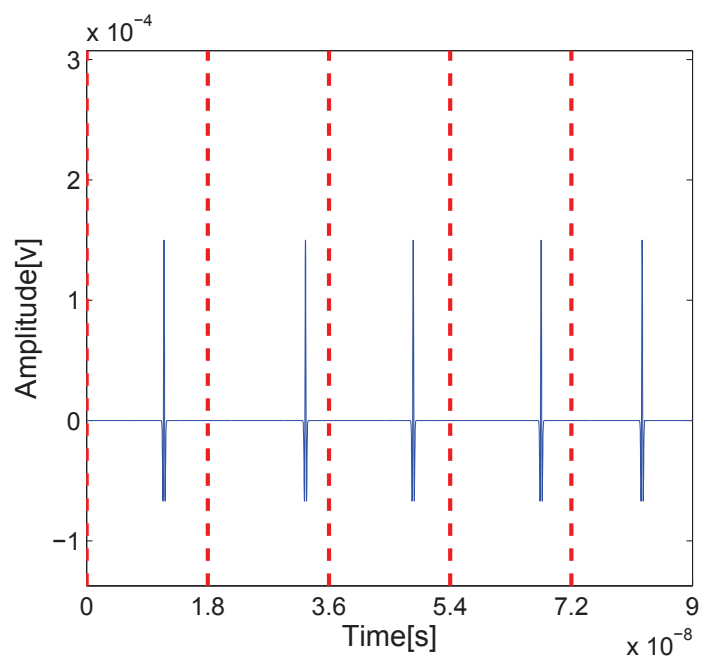

Fig. 9. Modification of PPM signalling to achieve maximum bit rate under the UWB hardware constraints. (Dashed lines indicate the symbol boundary)

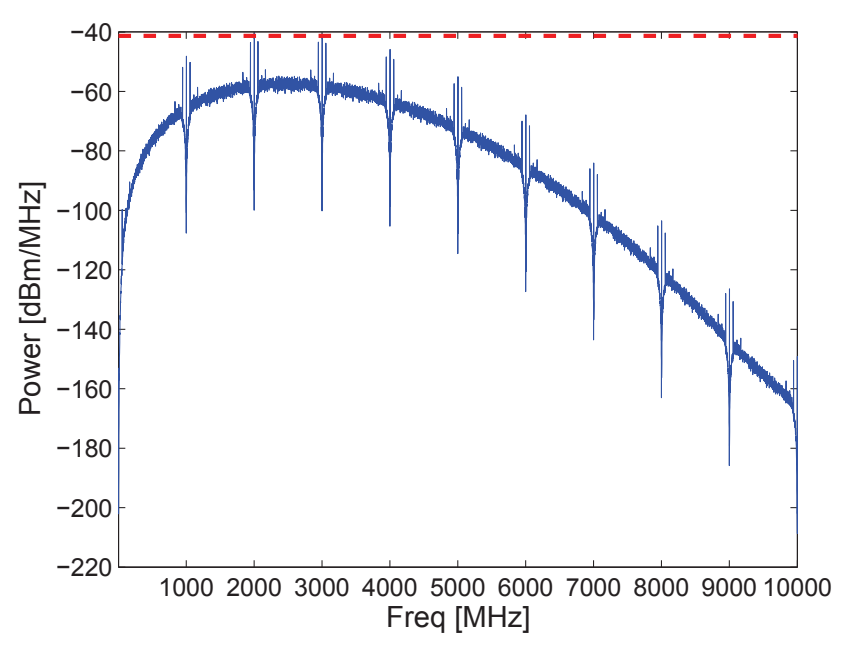

Fig. 10. PSD of PPM variant pulse train.

[2] L. E. Miller, "Why UWB? a review of ultra wideband technology," tech. rep., Wireless Communication Technology Group (WCTG) Report Gaitherburg, Maryland, April 2002.

[3] R. K. M. Ghavami, L.B. Michael, Ultra Wideband Signals and Systems in Communication Engineering. John Wiley, 2007.

[4] M. Ghavami, L. B. Michael, S. Haruyama, and R. Kohno, "A novel UWB pulse shape modulation system," Wireless Personal Communications, vol. 23, pp. 105-120, 2002. 10.1023/A:1020953424161.

[5] M. Win and R. Scholtz, "Impulse radio: how it works," Communications Letters, IEEE, vol. 2, pp. 36 -38, feb. 1998.

[6] K. Witrisal, G. Leus, G. Janssen, M. Pausini, F. Troesch, T. Zasowski, and J. Romme, "Noncoherent ultra-wideband systems," Signal Processing Magazine, IEEE, vol. 26, pp. 48 -66, july 2009.

[7] A. De Angelis, S. Dwivedi, and P. Händel, "A flexible UWB sensor for indoor localization," in Instrumentation and Measurement Technology Conference (I2MTC), 2012 IEEE International, pp. 2357 -2360, may 2012. 